MATHEMATICS OF COMPUTATION

Volume 76, Number 257, January 2007, Pages 97-114

S 0025-5718(06)01906-5

Article electronically published on September 28, 2006

\title{
A LEAST-SQUARES METHOD FOR SECOND ORDER NONCOERCIVE ELLIPTIC PARTIAL DIFFERENTIAL EQUATIONS
}

\author{
JAEUN KU
}

\begin{abstract}
In this paper, we consider a least-squares method proposed by Bramble, Lazarov and Pasciak (1998) which can be thought of as a stabilized Galerkin method for noncoercive problems with unique solutions. We modify their method by weakening the strength of the stabilization terms and present various new error estimates. The modified method has all the desirable properties of the original method; indeed, we shall show some theoretical properties that are not known for the original method. At the same time, our numerical experiments show an improvement of the method due to the modification.
\end{abstract}

\section{INTRODUCTION}

The existence and the uniqueness of the standard Galerkin finite element method for noncoercive problems with unique solutions were proved by Schatz [12] with the restriction of small meshsize $h$. It is easy to construct an example which does not admit a unique solution when the meshsize is not small enough. Thus, we can deduce that the restriction is real, not a limitation of the analysis used.

One of the problems concerning the small meshsize is that it is not possible to determine how small the meshsize should be to guarantee the existence and the uniqueness of the approximate solution without actually knowing the true solution.

There are many methods which guarantee the existence and the uniqueness of the approximate solution without the restriction on the meshsize. Most of them introduce a new variable and transform the original problem into a first-order system, which is then solved via a least-squares method. The known error estimates require relatively smooth solutions. We refer to [1, 2, 3, 4, 7, 8, 11] and further references therein. In [5], least-squares methods were considered directly on the second order problem.

Recently, Bramble, Lazarov and Pasciak [4 introduced and studied a new leastsquares method without a new variable involving a discrete computable $H^{-1}$-norm. This method could be thought of as a stabilized Galerkin method in the sense that stabilization terms are added to the Galerkin bilinear form to guarantee the existence and the uniqueness of the approximate solution without any restriction on the meshsize. The resulting linear algebraic system is symmetric and positive definite.

Received by the editor November 2, 2004 and, in revised form, July 7, 2005.

2000 Mathematics Subject Classification. Primary 65N30; Secondary 65N15.

Key words and phrases. Least-squares, stabilized Galerkin method, error estimates. Research supported in part by NSF grant DMS-0071412.

(C)2006 American Mathematical Society Reverts to public domain 28 years from publication 
In this paper, we observe that we can weaken the strength of the stabilization terms. As a result, we propose a new least-squares method. Since the stabilization terms are artificially added to produce a coercive bilinear form, it might be considered desirable to weaken the strength of the stabilization terms. Our numerical experiments show improvements due to the modification. Also, the new method has all the desirable theoretical properties of the original method, and we obtain various new error estimates including pointwise error estimates for our modified method.

This paper is organized as follows: In Section 2, we define notation, place assumptions on the problem which we are studying, and present some preliminary results. In Section 3 , we define a new least-squares method. In Section 4 and 5 we state and prove global and local error estimates based on the $L_{2}$-norm such as the energy norm. In Section 6, a pointwise error estimate is established. Finally, supporting numerical results are given in Section 7.

\section{Problem formulation AND PReliminaries}

Let $\Omega$ be a bounded convex polygonal or polyhedral domain in $\mathbb{R}^{n}$ where $n=2$ or 3. We shall consider the following second-order elliptic boundary problem:

$$
L u=f \quad \text { in } \Omega, \quad u=0 \quad \text { on } \partial \Omega .
$$

The operator $L$ is given by

$$
L u=-\triangle u+b \cdot \nabla u+c u=-\triangle u+\beta u .
$$

We assume that all the coefficients in the operator $L$ are smooth.

Remark. The results in this paper are true for $L u=-\operatorname{div}(\mathcal{A} \nabla u)+\beta u$, where the matrix $\mathcal{A}$ is symmetric, uniformly positive definite, and bounded.

We take $W=\stackrel{\circ}{H^{1}}(\Omega)$, and $H^{-1}(\Omega)$ will be the dual of $W$.

For any $u, v \in W$, we define the bilinear form

$$
A(u, v)=(\nabla u, \nabla v)+(\beta u, v),
$$

where $(\cdot, \cdot)$ is the usual $L_{2}$ inner product.

In this paper, we shall repeatedly use the fact that the bilinear form $A$ is bounded with respect to the norm in $H^{1}(\Omega)$.

The weak formulation of (2.1) is: Given $f \in H^{-1}(\Omega)$, find $u \in W$ satisfying

$$
A(u, \theta)=(f, \theta) \quad \text { for all } \theta \in W .
$$

The adjoint weak formulation of (2.1) is: Given $g \in H^{-1}(\Omega)$, find $w \in W$ satisfying

$$
A(\theta, w)=(g, \theta) \quad \text { for all } \theta \in W .
$$

We assume that the solutions of (2.4) and (2.5) are unique. Also, we assume that for $u$ and $w$ satisfying (2.4) and (2.5), respectively, there exists a positive constant $C$ independent of $f$ and $g$ satisfying

$$
\|u\|_{2} \leq C\|f\|_{0}, \quad\|w\|_{2} \leq C\|g\|_{0} .
$$

We give an alternative characterization of the norm in $H^{-1}(\Omega)$. Let $D(\cdot, \cdot)$ denote the inner product in $W$, i.e.,

$$
D(u, v)=(\nabla u, \nabla v)+(u, v) \quad \text { for all } u, v \in W .
$$


Let $T: H^{-1} \rightarrow W$ be defined by $T f=u$, where $u$ is the unique function satisfying $D(u, \theta)=(f, \theta)$ for all $\theta \in W$, i.e., $T$ is defined by

$$
D(T f, \theta)=(f, \theta) \quad \text { for all } \theta \in W .
$$

Then

$$
(u, T u)=\|u\|_{-1}^{2} \quad \text { for all } u \in H^{-1}(\Omega) .
$$

Note also that

$$
\|u\|_{i+1}=\|T f\|_{i+1} \leq C\|f\|_{i-1} \quad \text { for } i=0,1 .
$$

Let the operator $\mathcal{L}: W \rightarrow H^{-1}(\Omega)$ be defined by the identity

$$
(\mathcal{L} u, \phi)=A(u, \phi) \quad \text { for all } \phi \in W .
$$

To solve (2.1) approximately, we introduce the subspace $W_{h} \subset W$ indexed by $h$ in the interval $0<h<1$. We do this by partitioning the domain $\Omega$ into a set of triangles or tetrahedra $\mathcal{T}=\{\tau\}$. Let $h_{\tau}$ denote the diameter of the triangle $\tau$. The mesh parameter $h$ is defined to be

$$
h=\max _{\tau \in \mathcal{T}} h_{\tau} .
$$

We assume that the boundaries of two triangles or tetrahedra will intersect at either a vertex, an entire edge, or a face. We assume that the triangulations are globally quasi-uniform as defined in [6, (4.4.15)]. For some integer $2 \leq r$, let $W_{h}$ denote the functions which are piecewise polynomials of degree less than $r$ with respect to the triangles, continuous on $\Omega$, and vanish on $\partial \Omega$. There is a nodal basis associated with the spaces and a corresponding averaged nodal interpolation operator, 15.

The following low order approximation and boundedness result can be proved. Given $\phi \in W$, there exists $\phi_{I} \in W_{h}$ and a constant $C_{2}$ not dependent on $h$ and $\phi$ such that

$$
\sum_{\tau_{i} \in \mathcal{T}}\left\{h_{\tau_{i}}^{-2}\left\|\phi-\phi_{I}\right\|_{0, \tau_{i}}^{2}+\left\|\phi-\phi_{I}\right\|_{1, \tau_{i}}^{2}\right\} \leq C_{2}\|\phi\|_{1}^{2} .
$$

The argument which can be used to prove this result is given in [15.

The approximation space $W_{h}$ satisfies the following higher order approximation property:

$$
\sum_{\tau_{i} \in \mathcal{T}}\left(\left\|\phi-\phi_{I}\right\|_{o, \tau_{i}}^{2}+h_{\tau_{i}}^{2}\left\|\phi-\phi_{I}\right\|_{1, \tau_{i}}^{2}+\cdots+h^{2 r}\left\|\phi-\phi_{I}\right\|_{4, \tau_{i}}^{2}\right)^{1 / 2} \leq C h^{r}\|\phi\|_{r, \Omega}^{2} .
$$

Also, $W_{h}$ has the following approximation property based on the $L_{\infty}$-norm:

$$
\left\|\phi-\phi_{I}\right\|_{\infty} \leq C h\|\phi\|_{W_{\infty}^{1}} .
$$

We want to point out that the approximation space $W_{h}$ has the inverse property [6], i.e., for $1 \leq p \leq \infty, 1 \leq q \leq \infty$, and $0 \leq m \leq l$,

$$
\|\psi\|_{W_{p}^{l}(\tau)} \leq C h^{m-l+\frac{n}{p}-\frac{n}{q}}\|\psi\|_{W_{q}^{m}(\tau)} \quad \text { for all } \psi \in W_{h} \text {, for } n=2,3 .
$$

To describe the least-squares method, we shall need additional discrete norms and inner products. For $\psi \in W_{h}$, the discrete negative norm is given by

$$
\|\psi\|_{-1, h}=\sup _{\phi \in W_{h}} \frac{(\psi, \phi)}{\|\phi\|_{1}} .
$$

This norm extends to a semi-norm on $H^{-1}(\Omega)$ which is bounded by the norm $\|\cdot\|_{-1}$. 
Also, we define a new discrete norm as follows:

$$
\|v\|_{2}^{h}=\left(\sum_{\tau}\|v\|_{2, \tau}^{2}\right)^{1 / 2} .
$$

Let the operator $\mathcal{L}_{h}: W \rightarrow W_{h}$ be defined by

$$
\left(\mathcal{L}_{h} v, \phi\right)=A(v, \phi) \quad \text { for all } \phi \in W_{h} .
$$

Before describing the least-squares method, we provide an equivalent discrete negative norm. As in the continuous case, the discrete negative norm can be alternatively characterized in terms of a certain operator. Specifically, let $T_{h}: H^{-1}(\Omega) \rightarrow W_{h}$ be defined by

$$
D\left(T_{h} f, \theta\right)=(f, \theta) \quad \text { for all } \theta \in W_{h} .
$$

We state various properties which will be used later:

$$
\begin{gathered}
\left\|T_{h} f\right\|_{1} \leq C\|f\|_{-1}, \\
\left\|\left(T-T_{h}\right) f\right\|_{i} \leq C h^{2-i}\|f\|_{0} \quad \text { for } i=0,1 .
\end{gathered}
$$

Let $T_{h}$ be the finite element analogue of the operator $T$. Then

$$
\begin{aligned}
\|v\|_{-1, h}^{2}=\left(v, T_{h} v\right) & \text { for all } v \in H^{-1}(\Omega), \\
\left(v, T_{h} w\right)=\left(T_{h} v, w\right) & \text { for all } v, w \in L^{2}(\Omega) .
\end{aligned}
$$

For $\beta$ defined as in (2.2), we have

$$
\|\beta v\|_{-1} \leq C\|v\|_{0} \quad \text { for all } v \in W .
$$

By combining (2.16) and (2.20), we have

$$
\left\|T_{h} \beta v\right\|_{1} \leq C\|v\|_{0} \quad \text { for all } v \in W .
$$

The following lemmas play a fundamental role in the least-squares methods, and Lemmas 2.1 and 2.2 were proved in Bramble et al. 44.

Lemma 2.1 (4]). There exists a constant $C$ independent of $v \in W$ such that

$$
\|v\|_{1} \leq C \sup _{\phi \in W} \frac{A(v, \phi)}{\|\phi\|_{1}}=C\|\mathcal{L} v\|_{-1} \quad \text { and } \quad\|v\|_{1} \leq C \sup _{\phi \in W} \frac{A(\phi, v)}{\|\phi\|_{1}} .
$$

Lemma 2.2. There exists a positive constant $c$ not depending on $h$ such that for any $\psi \in W_{h}$

$$
c\|\psi\|_{1}^{2} \leq\left\|\mathcal{L}_{h} \psi\right\|_{-1, h}^{2}+\sum_{\tau} h_{\tau}^{2}(L \psi, L \psi)_{\tau}+\sum_{e} h_{\tau(e)} \int_{e}\left[\psi_{\nu}\right]^{2} d s,
$$

where $\sum_{e}$ is the summation over all interior edges (or faces) and $\left[\psi_{\nu}\right]$ denote the jump in the normal derivative $\psi_{\nu}$ across an interior edge.

We now improve the above inequality by weakening the strength of the last two terms.

Lemma 2.3. There exists a positive constant $c$ not depending on $h$ such that for any $\psi \in W_{h}$

$$
c\|\psi\|_{1}^{2} \leq\left\|\mathcal{L}_{h} \psi\right\|_{-1, h}^{2}+h^{2} \sum_{\tau} h_{\tau}^{2}(L \psi, L \psi)_{\tau}+h^{2} \sum_{e} h_{\tau(e)} \int_{e}\left[\psi_{\nu}\right]^{2} d s .
$$


Proof. By Lemma 2.1.

$$
c\|\psi\|_{1} \leq \sup _{v \in W} \frac{A(\psi, v)}{\|v\|_{1}} .
$$

We write $A(\psi, v)=A\left(\psi, v-v_{h}\right)+A\left(\psi, v_{h}\right)$, where $v_{h}$ is the Ritz projection with respect to $(\nabla, \nabla)$, i.e.,

$$
\left(\nabla\left(v-v_{h}\right), \nabla \psi\right)=0 \quad \text { for all } \psi \in W_{h} .
$$

Then using (2.14),

$$
\begin{aligned}
A(\psi, v) & =A\left(\psi, v-v_{h}\right)+A\left(\psi, v_{h}\right)=\left(\nabla \psi, \nabla\left(v-v_{h}\right)\right)+\left(\beta \psi, v-v_{h}\right)+\left(\mathcal{L}_{h} \psi, v_{h}\right) \\
& =\left(\beta \psi, v-v_{h}\right)+\left(\mathcal{L}_{h} \psi, v_{h}\right) .
\end{aligned}
$$

Using the above equality with $\left\|v_{h}\right\|_{1} \leq\|v\|_{1}$ and approximation property of $v_{h}$ in (2.22),

$$
c\|\psi\|_{1} \leq h\|\psi\|_{1}+\left\|\mathcal{L}_{h} \psi\right\|_{-1, h},
$$

i.e.,

$$
c\|\psi\|_{1}^{2} \leq\left\|\mathcal{L}_{h} \psi\right\|_{-1, h}^{2}+h^{2}\|\psi\|_{1}^{2} .
$$

By using Lemma 2.2 in the above inequality, we obtain

$$
c\|\psi\|_{1}^{2} \leq\left\|\mathcal{L}_{h} \psi\right\|_{-1, h}^{2}+h^{2} \sum_{\tau} h_{\tau}^{2}(L \psi, L \psi)_{\tau}+h^{2} \sum_{e} h_{\tau(e)} \int_{e}\left[\psi_{\nu}\right]^{2} d s .
$$

We now show a simple algebraic identity that will be of use in our development. It does not change the original method. This equality is used to prove error estimates.

Lemma 2.4. For any $v \in W, \psi \in W_{h}$,

$$
A(v, \psi)+A\left(v, T_{h}(\beta-1) \psi\right)=\left(\mathcal{L}_{h} v, T_{h} \mathcal{L}_{h} \psi\right) .
$$

In particular, for $\psi \in W_{h}$,

$$
A(\psi, \psi)+A\left(\psi, T_{h}(\beta-1) \psi\right)=\left(\mathcal{L}_{h} \psi, T_{h} \mathcal{L}_{h} \psi\right)=\left\|\mathcal{L}_{h} \psi\right\|_{-1, h}^{2} .
$$

Proof. By using that $T_{h}$ is symmetric (2.19), the definition of $\mathcal{L}_{h}$ in (2.14) and the property of $T_{h}$ in (2.15),

$$
\begin{aligned}
& \left(\mathcal{L}_{h} v, T_{h} \mathcal{L}_{h} \psi\right)=\left(\mathcal{L}_{h} \psi, T_{h} \mathcal{L}_{h} v\right)=A\left(\psi, T_{h} \mathcal{L}_{h} v\right)=\left(\nabla \psi, \nabla T_{h} \mathcal{L}_{h} v\right)+\left(\beta \psi, T_{h} \mathcal{L}_{h} v\right) \\
& \quad=\left(\psi, \mathcal{L}_{h} v\right)-\left(\psi, T_{h} \mathcal{L}_{h} v\right)+\left(\beta \psi, T_{h} \mathcal{L}_{h} v\right)=\left(\mathcal{L}_{h} v, \psi\right)-\left(\mathcal{L}_{h} v, T_{h} \psi\right)+\left(\mathcal{L}_{h} v, T_{h} \beta \psi\right) \\
& \quad=\left(\mathcal{L}_{h} v, \psi\right)+\left(\mathcal{L}_{h} v, T_{h} \beta \psi-T_{h} \psi\right)=A(v, \psi)+A\left(v, T_{h}(\beta-1) \psi\right) .
\end{aligned}
$$

By combining Lemmas 2.3 and 2.4, we obtain the following lemma.

Lemma 2.5. There exists a constant $c>0$ not depending on $h$ such that for any $\psi \in W_{h}$,

$$
c\|\psi\|_{1}^{2} \leq A(\psi, \psi)+A\left(\psi, T_{h}(\beta-1) \psi\right)+h^{2} \sum_{\tau} h_{\tau}^{2}(L \psi, L \psi)_{\tau}+h^{2} \sum_{e} h_{\tau(e)} \int_{e}\left[\psi_{\nu}\right]^{2} d s .
$$




\section{A LEAST-SQUARES FEM}

We define a new least-squares bilinear form on $W_{h} \times W_{h}$ by

$$
\begin{aligned}
B(\phi, \psi)=A(\phi, \psi) & +A\left(\phi, T_{h}(\beta-1) \psi\right) \\
& +h^{2} \sum_{\tau} h_{\tau}^{2}(L \phi, L \psi)_{\tau}+h^{2} \sum_{e} h_{\tau(e)} \int_{e}\left[\phi_{\nu}\right]\left[\psi_{\nu}\right] d s .
\end{aligned}
$$

Note that for $u \in W$ satisfying (2.1) with $f \in L^{2}$,

$$
B(u, \psi)=(f, \psi)+\left(f, T_{h}(\beta-1) \psi\right)+h^{2} \sum_{\tau} h_{\tau}^{2}(f, L \psi)_{\tau} \quad \text { for all } \psi \in W_{h} .
$$

Define the approximate solution $u_{h} \in W_{h}$ by

$$
B\left(u_{h}, \psi\right)=(f, \psi)+\left(f, T_{h}(\beta-1) \psi\right)+h^{2} \sum_{\tau} h_{\tau}^{2}(f, L \psi)_{\tau} \quad \text { for all } \psi \in W_{h} .
$$

By Lemma 2.5, $B(\cdot, \cdot)$ is coercive on $W_{h} \times W_{h}$. Hence the approximate solution is uniquely determined.

We have the orthogonal property as follows:

$$
B\left(u-u_{h}, \psi\right)=0 \text { for all } \psi \in W_{h} .
$$

Before we move on to our error analysis, we want to remark on the inequality given in Lemma 2.5. We use the following well-known trace inequality:

$$
\int_{\partial \tau}|\theta|^{2} d s \leq C\left(\frac{1}{h_{\tau}}\|\theta\|_{\tau}^{2}+h_{\tau}\|\theta\|_{1, \tau}^{2}\right) .
$$

By using Lemma 2.5 twice, the above trace inequality, and the inverse inequality in (2.11),

$$
\begin{aligned}
c\|\psi\|_{1}^{2} \leq & A(\psi, \psi)+A\left(\psi, T_{h}(\beta-1) \psi\right) \\
& +h^{2} \sum_{\tau} h_{\tau}^{2}(L \psi, L \psi)_{\tau}+h^{2} \sum_{e} h_{\tau(e)} \int_{e}\left[\psi_{\nu}\right]^{2} d s \\
\leq & A(\psi, \psi)+A\left(\psi, T_{h}(\beta-1) \psi\right)+C h^{2}\|\psi\|_{1}^{2} \\
\leq & A(\psi, \psi)+A\left(\psi, T_{h}(\beta-1) \psi\right) \\
& +\frac{C}{c} h^{2}\left(A(\psi, \psi)+A\left(\psi, T_{h}(\beta-1) \psi\right)\right. \\
& \left.\quad+h^{2} \sum_{\tau} h_{\tau}^{2}(L \psi, L \psi)_{\tau}+h^{2} \sum_{e} h_{\tau(e)} \int_{e}\left[\psi_{\nu}\right]^{2} d s\right) \\
\leq & \left(1+h^{2} \frac{C}{c}\right)\left(A(\psi, \psi)+A\left(\psi, T_{h}(\beta-1) \psi\right)\right. \\
& \left.+h^{4} \sum_{\tau} h_{\tau}^{2}(L \psi, L \psi)_{\tau}+h^{4} \sum_{e} h_{\tau(e)} \int_{e}\left[\psi_{\nu}\right]^{2} d s\right),
\end{aligned}
$$

i.e.,

$$
\begin{aligned}
\left(\frac{c}{1+h^{2} \frac{C}{c}}\right)\|\psi\|_{1}^{2} \leq A(\psi, \psi)+ & A\left(\psi, T_{h}(\beta-1) \psi\right) \\
& +h^{4} \sum_{\tau} h_{\tau}^{2}(L \psi, L \psi)_{\tau}+h^{4} \sum_{e} h_{\tau(e)} \int_{e}\left[\psi_{\nu}\right]^{2} d s .
\end{aligned}
$$


We can iterate the above argument as many times as we want. For each iteration, while we have a higher power of $h$ on the right-hand side, we have a smaller constant on the left-hand side. This suggests that we will have bigger numbers in the error as powers of $h$ are getting bigger. We present numerics supporting this in Section 7.

Our analysis is based on Lemma 2.5 for simplicity.

\section{Global error estimate}

The following theorem is essentially proved in [4].

Theorem 4.1. Let $u \in H^{\gamma}(\Omega)$ for $2 \leq \gamma \leq r$ and let $u_{h}$ be the solution of the leastsquares method defined by (3.2). Assume that the triangulation is quasi-uniform and $h=\max _{i} h_{\tau_{i}}$. Then there exists a positive constant $C$ not depending on $u$ or $h$ such that

$$
\left\|u-u_{h}\right\|_{1} \leq C\left(\left\|u-u_{I}\right\|_{1}+h\left\|u-u_{I}\right\|_{2}^{h}\right) \leq C h^{\gamma-1}\|u\|_{\gamma} .
$$

The following lemma is an immediate consequence of the triangle inequality, the inverse inequality (2.11), and Theorem 4.1 .

Lemma 4.2. Under the same assumption as Theorem 4.1,

$$
h^{2}\left\|u-u_{h}\right\|_{2}^{h} \leq C h\left(\left\|u-u_{I}\right\|_{1}+h\left\|u-u_{I}\right\|_{2}^{h}\right) \leq C h^{\gamma}\|u\|_{\gamma} .
$$

We now state and prove $L^{2}(\Omega)$ and $H^{-1}(\Omega)$ error estimates for the least-squares method in the case of a quasi-uniform mesh and sufficiently regular solutions. We want to point out that this is a new error estimate for this type of method.

Theorem 4.3. Under the same assumptions as Theorem 4.1,

$$
\left\|u-u_{h}\right\|_{0} \leq C h^{\gamma}\|u\|_{\gamma} .
$$

Proof. We have

$$
\left\|u-u_{h}\right\|_{0}=\sup _{\phi \in L_{2},\|\phi\|_{0}=1}\left(u-u_{h}, \phi\right) .
$$

For each such $\phi$, let $v$ be the solution of the adjoint problem:

$$
A(z, v)=(z, \phi) \quad \text { for all } z \in W .
$$

By taking $z=u-u_{h}$,

$$
\left(u-u_{h}, \phi\right)=A\left(u-u_{h}, v\right) .
$$

Let $w$ be such that

$$
\begin{aligned}
-\triangle w+\beta w & =-\Delta v+v & & \text { in } \Omega, \\
w & =0 & & \text { on } \partial \Omega .
\end{aligned}
$$

By (2.6),

$$
\|w\|_{2} \leq C\|-\triangle v+v\|_{0} \leq C\|v\|_{2} \leq C\|\phi\|_{0} \leq C .
$$

By applying $T$ to both sides of (4.2), we get

$$
w+T(\beta-1) w=v .
$$

Hence,

$$
\begin{aligned}
\left(u-u_{h}, \phi\right)= & A\left(u-u_{h}, v\right)=A\left(u-u_{h}, w\right)+A\left(u-u_{h}, T(\beta-1) w\right) \\
= & A\left(u-u_{h}, w\right)+A\left(u-u_{h}, T_{h}(\beta-1) w\right) \\
& +A\left(u-u_{h},\left(T-T_{h}\right)(\beta-1) w\right) .
\end{aligned}
$$


For the first two terms on the right, by using the orthogonal property (3.3),

$$
\begin{aligned}
A(u & \left.-u_{h}, w\right)+A\left(u-u_{h}, T_{h}(\beta-1) w\right) \\
& =A\left(u-u_{h}, w-w_{I}\right)+A\left(u-u_{h}, T_{h}(\beta-1) w-w_{I}\right) \\
& -h^{2} \sum_{\tau} h_{\tau}^{2}\left(L\left(u-u_{h}\right), L\left(w_{I}\right)\right)_{\tau}+h^{2} \sum_{e} h_{\tau(e)} \int_{e}\left[\left(u-u_{h}\right)_{\nu}\right]\left[\left(-w_{I}\right)_{\nu}\right] d s \\
& =I_{1}+I_{2}+I_{3}+I_{4},
\end{aligned}
$$

where $w_{I}$ is the interpolant 15 of $w$ in $W_{h}$. We then have

$$
\begin{aligned}
\left|I_{1}\right| & =\left|A\left(u-u_{h}, w-w_{I}\right)\right| \leq C\left\|u-u_{h}\right\|_{1}\left\|w-w_{I}\right\|_{1} \\
& \leq C h\left\|u-u_{h}\right\|_{1}\|w\|_{2} \leq C h\left\|u-u_{h}\right\|_{1} \leq C h^{\gamma}\|u\|_{\gamma} .
\end{aligned}
$$

By using (2.21),

$$
\begin{aligned}
\left|I_{2}\right| & =\left|A\left(u-u_{h}, T_{h}(\beta-1)\left(w-w_{I}\right)\right)\right| \leq C\left\|u-u_{h}\right\|_{1}\left\|T_{h}(\beta-1)\left(w-w_{I}\right)\right\|_{1} \\
& \leq C\left\|u-u_{h}\right\|_{1}\left\|w-w_{I}\right\|_{0} \leq C h^{\gamma+1}\|u\|_{\gamma} .
\end{aligned}
$$

By $\left\|w_{I}\right\|_{2}^{h} \leq C\|w\|_{2} \leq C$ and Lemma 4.2 .

$$
\begin{aligned}
\left|I_{3}\right| & =\left|h^{2} \sum_{\tau} h_{\tau}^{2}\left(L\left(u-u_{h}\right), L w_{I}\right)_{\tau}\right| \leq C h^{4} \sum_{\tau}\left\|u-u_{h}\right\|_{2, \tau}\left\|w_{I}\right\|_{2, \tau} \\
& \leq C h^{4}\left\|u-u_{h}\right\|_{2}^{h}\|w\|_{2} \leq C h^{\gamma+2}\|u\|_{\gamma}\|w\|_{2} \leq C h^{\gamma+2}\|u\|_{\gamma} .
\end{aligned}
$$

By using $\left[w_{\nu}\right]=0$, the trace inequality (3.4), (2.9), Lemma 4.2, and Theorem 4.1,

$$
\begin{aligned}
\left|I_{4}\right| \leq & \left|h^{2} \sum_{e} h_{\tau(e)} \int_{e}\left[\left(u-u_{h}\right)_{\nu}\right]\left[\left(w-w_{I}\right)_{\nu}\right] d s\right| \\
\leq & C h^{3} \sum_{e}\left(\int_{e}\left[\left(u-u_{h}\right)_{\nu}\right]^{2} d s\right)^{1 / 2}\left(\int_{e}\left[\left(w-w_{I}\right)_{\nu}\right]^{2} d s\right)^{1 / 2} \\
\leq & C h^{3} \sum_{\tau}\left(\frac{1}{\sqrt{h}}\left\|u-u_{h}\right\|_{1, \tau}+\sqrt{h}\left\|u-u_{h}\right\|_{2, \tau}\right) \\
& \cdot\left(\frac{1}{\sqrt{h}}\left\|w-w_{I}\right\|_{1, \tau}+\sqrt{h}\left\|w-w_{I}\right\|_{2, \tau}\right) \\
\leq & C h^{3} \sum_{\tau}\left(\frac{1}{\sqrt{h}}\left\|u-u_{h}\right\|_{1, \tau}+\sqrt{h}\left\|u-u_{h}\right\|_{2, \tau}\right)\left(\sqrt{h}\|w\|_{2, \tau}\right) \\
\leq & C h^{3} \sum_{\tau}\left(\left\|u-u_{h}\right\|_{1, \tau}+h\left\|u-u_{h}\right\|_{2, \tau}\right)\|w\|_{2, \tau} \\
\leq & C h^{3}\left(\left\|u-u_{h}\right\|_{1}+h\left\|u-u_{h}\right\|_{2}^{h}\right)\|w\|_{2} \\
\leq & C h^{\gamma+2}\|u\|_{\gamma} .
\end{aligned}
$$

Thus,

$$
\left|A\left(u-u_{h}, w\right)+A\left(u-u_{h}, T_{h}(\beta-1) w\right)\right| \leq C h^{\gamma}\|u\|_{\gamma} .
$$

Using (2.17) and (2.21) for the third term on the right in (4.4),

$$
\begin{aligned}
\left|A\left(u-u_{h},\left(T-T_{h}\right)(\beta-1) w\right)\right| & \leq C\left\|u-u_{h}\right\|_{1}\left\|\left(T-T_{h}\right)(\beta-1) w\right\|_{1} \\
& \leq C h\left\|u-u_{h}\right\|_{1}\|w\|_{1} \leq C h^{\gamma}\|u\|_{\gamma} .
\end{aligned}
$$

By putting (4.5) and (4.6) into (4.4), we get

$$
\left(u-u_{h}, \phi\right) \leq C h^{\gamma}\|u\|_{\gamma} .
$$


Hence, we conclude that

$$
\left\|u-u_{h}\right\|_{0} \leq C h^{\gamma}\|u\|_{\gamma} .
$$

As for a negative norm estimate, we have the following.

Theorem 4.4. With the same assumptions as above and $r \geq 3$ and $\Omega$ bounded in $\mathbb{R}^{2}$

$$
\left\|u-u_{h}\right\|_{-1} \leq C h^{\gamma-1+\alpha}\|u\|_{\gamma},
$$

where $\alpha=\min \left\{2, \frac{\pi}{\theta}-\epsilon\right\}$ with $\theta$ the largest angle in $\Omega$ and $\epsilon>0$.

Note that the solution to (2.1) belongs to $H^{1+\alpha}$ where $\alpha$ is defined as above; cf. [9.

Proof.

$$
\left\|u-u_{h}\right\|_{-1}=\sup _{\phi \in W,\|\phi\|_{1}=1}\left(u-u_{h}, \phi\right) .
$$

The remaining proof is similar to that of Theorem 4.3

\section{LOCAL ERROR ESTIMATES}

This section is motivated by the work of Nitche and Schatz [10]. Rather than following the proof given in [10, we use the slightly varied argument given by Wahlbin [17. The importance of the local $H^{1}$ error estimate is that it is essential for our pointwise error estimates in Section 6 .

Theorem 5.1. Let $D \subset \Omega, \widetilde{D}_{d}=\left\{x \in \mathbb{R}^{n}: \operatorname{dist}(x, D) \leq d\right\}$, and $D_{d}=\Omega \cap \widetilde{D}_{d}$ with $d \geq C_{0} h$ for $C_{0}$ sufficiently large. With the same assumptions as in Theorem 4.1 ,

$$
\begin{aligned}
\left\|u-u_{h}\right\|_{1, D} \leq & C\left(\|u-\chi\|_{1, D_{d}}+\frac{\|u-\chi\|_{0, D_{d}}}{d}+\frac{\left\|u-u_{h}\right\|_{0, D_{d}}}{d}\right) \\
& +C\left(h^{3}\|u-\chi\|_{2, D_{d}}^{h}+h\|u-\chi\|_{1, \Omega}+\left\|u-u_{h}\right\|_{0, \Omega}+\|u-\chi\|_{0, \Omega}\right),
\end{aligned}
$$

for any $\chi \in W_{h}$.

The first three terms on the right in the above inequality are exactly the same as the terms in 10 . The last four terms result from the stabilization terms, and some of them involve the global norm. We want to point out that they are "weaker" terms compared to the first three terms.

Proof. We first note that the desired estimate follows if we can show that

$$
\begin{aligned}
\left\|\nabla\left(u-u_{h}\right)\right\|_{0, D} \leq & C\left(\|u\|_{1, D_{d}}+\frac{\|u\|_{0, D_{d}}}{d}+\frac{\left\|u-u_{h}\right\|_{0, D_{d}}}{d}\right) \\
& +C\left(h^{3}\|u\|_{2, D_{d}}^{h}+h\|u\|_{1, \Omega}+\left\|u-u_{h}\right\|_{0, \Omega}+\|u\|_{0, \Omega}\right) ;
\end{aligned}
$$

then just write $u-u_{h}=(u-\chi)+\left(\chi-u_{h}\right)$ for any $\chi \in W_{h}$. By the triangle inequality, the above inequality in turn follows if we can show that

$$
\begin{aligned}
\left\|\nabla u_{h}\right\|_{0, D} \leq & C\left(\|u\|_{1, D_{d}}+\frac{\left\|u_{h}\right\|_{0, D_{d}}}{d}\right) \\
& +C\left(h^{3}\|u\|_{2, D_{d}}^{h}+h\|u\|_{1, \Omega}+\left\|u_{h}\right\|_{0, \Omega}+\|u\|_{0, \Omega}\right) .
\end{aligned}
$$


Now let $\omega$ be a cut-off function which equals 1 on $D$, is supported in $\widetilde{D}_{\frac{d}{2}}$, and satisfies $|\omega|_{W_{\infty}^{l}} \leq C d^{-l}$, where $l=0,1,2$. Then, by definition of $A(\cdot, \cdot)$,

$$
\begin{aligned}
\left\|\nabla u_{h}\right\|_{0, D}^{2} \leq & \left\|\omega \nabla u_{h}\right\|_{0, D_{d}}^{2}=\left(\omega \nabla u_{h}, \omega \nabla u_{h}\right) \\
= & A\left(u_{h}, \omega^{2} u_{h}\right)-\int\left(b \cdot \nabla u_{h}\right) \omega^{2} u_{h}+c(x) \omega^{2} u_{h}^{2} d x \\
& -2 \int\left(\omega \nabla u_{h}\right) \cdot\left(u_{h} \nabla \omega\right) d x \\
= & A\left(u_{h}-u, \omega^{2} u_{h}\right)-\int\left(b \cdot \nabla u_{h}\right) \omega^{2} u_{h}+c(x) \omega^{2} u_{h}^{2} d x \\
& -2 \int\left(\omega \nabla u_{h}\right) \cdot\left(u_{h} \nabla \omega\right) d x+A\left(u, \omega^{2} u_{h}\right) \\
= & I_{1}+I_{2}+I_{3}+I_{4}+I_{5} .
\end{aligned}
$$

For $I_{1}$, by the orthogonal property (3.3),

$$
\begin{aligned}
I_{1}= & A\left(u_{h}-u, \omega^{2} u_{h}\right) \\
= & A\left(u_{h}-u, \omega^{2} u_{h}-\psi\right)-A\left(u_{h}-u, T_{h}(\beta-1) \psi\right) \\
& -h^{2} \sum_{\tau} h_{\tau}^{2}\left(L\left(u_{h}-u\right), L \psi\right)_{\tau}-h^{2} \sum_{e} h_{\tau(e)} \int_{e}\left[\left(u_{h}-u\right)_{\gamma}\right]\left[\psi_{\gamma}\right] d s \\
= & J_{1}+J_{2}+J_{3}+J_{4},
\end{aligned}
$$

where $\psi=\left(\omega^{2} u_{h}\right)_{I}$, i.e., the interpolant defined in [15].

We state some of the inequalities needed. Note that the second inequality is the superapproximation property; cf. [10. The proof can be obtained by using Poincaré inequality and differentiating $\psi$ :

$$
\begin{gathered}
\|\psi\|_{1} \leq \frac{C}{d}\left\|u_{h}\right\|_{0, D_{d}}+C\left\|\omega \nabla u_{h}\right\|_{0, D_{d}}, \\
\left\|\omega^{2} u_{h}-\psi\right\|_{1} \leq C\left(\frac{h}{d}\left\|u_{h}\right\|_{1, D_{d}}+\frac{h}{d^{2}}\left\|u_{h}\right\|_{0, D_{d}}\right) .
\end{gathered}
$$

For $J_{1}$, using (5.4) and (2.11),

$$
\begin{aligned}
\left|J_{1}\right| & =\left|A\left(u_{h}-u, \omega^{2} u_{h}-\psi\right)\right| \leq C\left\|u-u_{h}\right\|_{1, D_{d}}\left\|\omega^{2} u_{h}-\psi\right\|_{1} \\
& \leq C\left(\|u\|_{1, D_{d}}+\left\|u_{h}\right\|_{1, D_{d}}\right)\left(\frac{h}{d}\left\|u_{h}\right\|_{1, D_{d}}+\frac{h}{d^{2}}\left\|u_{h}\right\|_{0, D_{d}}^{2}\right) \\
& \leq C\|u\|_{1, D_{d}}^{2}+\frac{\left\|u_{h}\right\|_{0, D_{d}}^{2}}{d^{2}}+\frac{h}{d}\left\|u_{h}\right\|_{1, D_{d}}^{2} .
\end{aligned}
$$

By the definition of $T$ (2.7),

$$
\begin{aligned}
J_{2}= & A\left(u-u_{h}, T_{h}(\beta-1) \psi\right) \\
= & \left(\nabla\left(u-u_{h}\right), \nabla\left(T_{h}-T\right)(\beta-1) \psi\right)+\left(\beta\left(u-u_{h}\right), T_{h}(\beta-1) \psi\right) \\
& \quad+\left(\nabla\left(u-u_{h}\right), \nabla T(\beta-1) \psi\right) \\
= & \left(\nabla\left(u-u_{h}\right), \nabla\left(T_{h}-T\right)(\beta-1) \psi\right)+\left(\beta\left(u-u_{h}\right), T_{h}(\beta-1) \psi\right) \\
& \quad+\left(u-u_{h},(\beta-1) \psi\right)-\left(u-u_{h}, T(\beta-1) \psi\right) .
\end{aligned}
$$


Thus, using (2.17), (5.3), and (2.11),

$$
\begin{aligned}
\left|J_{2}\right| \leq & \left\|u-u_{h}\right\|_{1, \Omega}\left\|\left(T-T_{h}\right)(\beta-1) \psi\right\|_{1, \Omega}+\left\|u-u_{h}\right\|_{1, \Omega}\left\|T_{h}(\beta-1) \psi\right\|_{0, \Omega} \\
& +\left\|u-u_{h}\right\|_{0, \Omega}\|(\beta-1) \psi\|_{0, \Omega}+\left\|u-u_{h}\right\|_{0, \Omega}\|T(\beta-1) \psi\|_{0, \Omega} \\
\leq & C h\left\|u-u_{h}\right\|_{1, \Omega}\|\psi\|_{1}+C\left\|u-u_{h}\right\|_{0, \Omega}\|\psi\|_{1} \\
\leq & C\left(h\|u\|_{1, \Omega}+\left\|u_{h}\right\|_{0, \Omega}\right)\left(\frac{\left\|u_{h}\right\|_{0, D_{d}}}{d}+\left\|\omega \nabla u_{h}\right\|_{0, D_{d}}\right) \\
& +C\left(\|u\|_{0, \Omega}+\left\|u_{h}\right\|_{0, \Omega}\right)\left(\frac{\left\|u_{h}\right\|_{0, D_{d}}}{d}+\left\|\omega \nabla u_{h}\right\|_{0, D_{d}}\right) \\
\leq & C\left(h^{2}\|u\|_{1, \Omega}^{2}+\left\|u_{h}\right\|_{0, \Omega}^{2}+\frac{\left\|u_{h}\right\|_{0, D_{d}}^{2}}{d^{2}}+\|u\|_{0, \Omega}^{2}\right)+\frac{1}{C}\left\|\omega \nabla u_{h}\right\|_{0, D_{d}}^{2} .
\end{aligned}
$$

Using that $\operatorname{supp}(\psi) \subset D_{d}$ and by (2.11),

$$
\begin{aligned}
\left|J_{3}\right| & =\left|h^{2} \sum_{\tau} h_{\tau}^{2}\left(L\left(u-u_{h}\right), L \psi\right)_{\tau}\right| \leq C h^{4} \sum_{\tau \subset D_{d}}\left\|u-u_{h}\right\|_{2, \tau}\|\psi\|_{2, \tau} \\
& \leq C h^{3} \sum_{\tau \subset D_{d}}\left\|u-u_{h}\right\|_{2, \tau}\|\psi\|_{1, \tau} \leq C h^{3}\left\|u-u_{h}\right\|_{2, D_{d}}^{h}\|\psi\|_{1} \\
& \leq C h^{3}\left(\|u\|_{2, D_{d}}^{h}+\left\|u_{h}\right\|_{2, D_{d}}^{h}\right)\left(\frac{\left\|u_{h}\right\|_{0, D_{d}}}{d}+\left\|\omega \nabla u_{h}\right\|_{0, D_{d}}\right) \\
& \leq C\left(\left(h^{3}\|u\|_{2, D_{d}}^{h}\right)^{2}+\left\|u_{h}\right\|_{0, D_{d}}^{2}\right)+\frac{1}{C}\left\|\omega \nabla u_{h}\right\|_{0, D_{d}}^{2} .
\end{aligned}
$$

By the trace inequality (3.4), the inverse inequality (2.11), the superapproximation property (5.3), and the arithmetic-geometric mean inequality,

$$
\begin{aligned}
\left|J_{4}\right| & =\left|h^{2} \sum_{e} h_{\tau(e)} \int_{e}\left[\left(u-u_{h}\right)_{\nu}\right]\left[\psi_{\nu}\right] d s\right| \\
& \leq C h^{3} \sum_{e \subset D_{d}}\left(\int_{e}\left[\left(u-u_{h}\right)_{\gamma}\right]^{2} d s\right)^{1 / 2}\left(\int_{e}\left[\psi_{\nu}\right]^{2} d s\right)^{1 / 2} \\
& \leq C h^{3} \sum_{\tau \subset D_{d}}\left(\left(\frac{1}{\sqrt{h}}\left\|u-u_{h}\right\|_{1, \tau}+\sqrt{h}\left\|u-u_{h}\right\|_{2, \tau}\right)\left(\frac{1}{\sqrt{h}}\|\psi\|_{1, \tau}+\sqrt{h}\|\psi\|_{2, \tau}\right)\right) \\
& \leq C h^{2} \sum_{\tau \subset D_{d}}\left(\left\|u-u_{h}\right\|_{1, \tau}+h\left\|u-u_{h}\right\|_{2, \tau}\right)\left(\|\psi\|_{1, \tau}\right) \\
& \leq C h^{2}\left(\left\|u-u_{h}\right\|_{1, D_{d}}+h\left\|u-u_{h}\right\|_{2, D_{d}}^{h}\right)\|\psi\|_{1} \\
& \leq C\left(h^{2}\|u\|_{1, D_{d}}+h^{3}\|u\|_{2, D_{d}}^{h}+h^{2}\left\|u_{h}\right\|_{1, D_{d}}\right)\left(\frac{\left\|u_{h}\right\|_{0, D_{d}}}{d}+\left\|\omega \nabla u_{h}\right\|_{0, D_{d}}\right) \\
& \leq C\left(h^{4}\|u\|_{1, D_{d}}^{2}+\left(h^{3}\|u\|_{2, D_{d}}^{h}\right)^{2}+\frac{\left\|u_{h}\right\|_{0, D_{d}}^{2}}{d^{2}}\right)+\frac{1}{C}\left\|\omega \nabla u_{h}\right\|_{0, D_{d}}^{2} .
\end{aligned}
$$


Thus

$$
\begin{aligned}
\left|I_{1}\right| \leq & C\left(\|u\|_{1, D_{d}}^{2}+\frac{\left\|u_{h}\right\|_{0, D_{d}}^{2}}{d^{2}}\right) \\
& +C\left(h^{2}\|u\|_{1, \Omega}^{2}+\|u\|_{0, \Omega}^{2}+\left\|u_{h}\right\|_{0, \Omega}^{2}+\left(h^{3}\|u\|_{2, D_{d}}^{h}\right)^{2}\right) \\
& +C \frac{h}{d}\left\|u_{h}\right\|_{1, D_{d}}^{2}+\frac{1}{C}\left\|\omega \nabla u_{h}\right\|_{0, D_{d}}^{2} .
\end{aligned}
$$

Using the arithmetic-geometric mean inequality, we can easily obtain the following inequality:

$$
\left|I_{2}\right|+\left|I_{3}\right|+\left|I_{4}\right|+\left|I_{5}\right| \leq C\left(\|u\|_{1, D_{d}}^{2}+\frac{\left\|u_{h}\right\|_{0, D_{d}}^{2}}{d^{2}}\right)+\frac{1}{C}\left\|\omega \nabla u_{h}\right\|_{0, D_{d}}^{2} .
$$

By putting the estimates for $I_{1}, I_{2}, I_{3}, I_{4}$, and $I_{5}$ into (5.2) and using a kick-back argument with sufficiently large $C$, we can conclude that

$$
\begin{aligned}
\left\|\nabla u_{h}\right\|_{0, D}^{2} \leq & C\left(\|u\|_{1, D_{d}}^{2}+\frac{\left\|u_{h}\right\|_{0, D_{d}}^{2}}{d^{2}}\right) \\
& +C\left(h^{2}\|u\|_{1, \Omega}^{2}+\|u\|_{0, \Omega}^{2}+\left\|u_{h}\right\|_{0, \Omega}^{2}+\left(h^{3}\|u\|_{2, D_{d}}^{h}\right)^{2}\right) \\
& +C \frac{h}{d}\left\|u_{h}\right\|_{1, D_{d}}^{2} .
\end{aligned}
$$

Iterating the above argument for $\left\|\nabla u_{h}\right\|_{0, D_{d}}^{2}$ with $D_{2 d}$ and using the inverse inequality (2.11), we get

$$
\begin{aligned}
\left\|\nabla u_{h}\right\|_{0, D}^{2} \leq & C\left(\|u\|_{1, D_{2 d}}^{2}+\frac{\left\|u_{h}\right\|_{0, D_{2 d}}^{2}}{(2 d)^{2}}\right) \\
& +C\left(h^{2}\|u\|_{1, \Omega}^{2}+\|u\|_{0, \Omega}^{2}+\left\|u_{h}\right\|_{0, \Omega}^{2}+\left(h^{3}\|u\|_{2, D_{2 d}}^{h}\right)^{2}\right) \\
& +C \frac{1}{(2 d)^{2}}\left\|u_{h}\right\|_{0, D_{2 d}}^{2} .
\end{aligned}
$$

Now changing the notation back from $2 d$ to $d$, we obtain the inequality (5.1). Hence the theorem is proved.

\section{Pointwise error estimates}

From now on, our space will be in $\mathbb{R}^{2}$, i.e., $n=2$. We will closely follow the work of Schatz and Wahlbin 14 for the standard Galerkin method. However, the use of the nonlocal operator $T_{h}$ in our present method complicates the analysis.

Without loss of generality we assume that $\operatorname{diam}(\Omega) \leq 1$. Let

$$
d_{j}=2^{-j} \quad \text { for } j=0,1,2, \ldots,
$$

and for fixed $x$ set

$$
\begin{aligned}
& \Omega_{j}=\left\{y \in \Omega: d_{j+1}<|y-x|<d_{j}\right\} \\
& \Omega_{j}^{\prime}=\left\{y \in \Omega: d_{j+2}<|y-x|<d_{j-1}\right\}, \\
& \Omega_{j}^{\prime \prime}=\left\{y \in \Omega: d_{j+3}<|y-x|<d_{j-2}\right\} .
\end{aligned}
$$

We shall now define two functions $g^{x}(y)$ and $g_{h}^{x}(y)$. Here $g^{x}(y)$ may be thought of as a smoothed Green's function with a singularity at $x$, and $g_{h}^{x}(y) \in W_{h}$ its finite element approximation. We now give some facts that will be needed for the proof 
of pointwise estimates. For $d>0$ and any fixed $x \in \bar{\Omega}, B_{d}(x)$ will denote the intersection of $\Omega$ with a ball of radius $d$ centered at $x$, i.e.,

$$
B_{d}(x)=\{y \in \Omega:\|y-x\|<d\} .
$$

Define

$$
\eta= \begin{cases}h^{-1} \frac{\left(u-u_{h}\right)(y)}{\left\|u-u_{h}\right\|_{L_{2}\left(B_{2 h}(x)\right)}} & \text { if } y \in B_{2 h}(x), \\ 0 & \text { otherwise. }\end{cases}
$$

For fixed $x \in \bar{\Omega}, g^{x}(y)$ is defined to satisfy

$$
A\left(v, g^{x}\right)=(\eta, v) \quad \text { for all } v \in W .
$$

By the a priori estimates (2.6),

$$
\left\|g^{x}\right\|_{W_{2}^{2}(\Omega)} \leq C\|\eta\|_{L_{2}(\Omega)} \leq \frac{C}{h}
$$

The finite element approximation $g_{h}^{x}(y) \in W_{h}$ is taken to be the unique solution of

$$
B\left(\phi, g^{x}-g_{h}^{x}\right)=0 \quad \text { for all } \phi \in W_{h} .
$$

We state and prove two inequalities concerning $g^{x}$ and $g_{h}^{x}$, which will be used later.

Lemma 6.1. Let $g^{x}$ satisfy (6.2). Then

$$
\left\|g^{x}\right\|_{1} \leq C \ln \frac{1}{h}
$$

Proof. By the a priori estimate (2.6), $\left\|g^{x}\right\|_{1} \leq C\|\eta\|_{-1}$. By definition,

$$
\|\eta\|_{-1}=\sup _{\phi \in W,\|\phi\|_{1}=1}(\eta, \phi) .
$$

By Hölder's inequality,

$$
(\eta, \phi) \leq\|\eta\|_{L_{q}}\|\phi\|_{L_{p}}, \quad \text { where } \frac{1}{p}+\frac{1}{q}=1 .
$$

By Sobolev's inequality,

$$
\|\phi\|_{L_{p}} \leq C p\|\phi\|_{1}=C p
$$

so that

$$
(\eta, \phi) \leq C p\|\eta\|_{L_{q}} .
$$

Choose $p=\ln \frac{1}{h}$. Again, by using Hölder's inequality, the fact that $\operatorname{supp}(\eta) \subset$ $B_{2 h}(x)$, and choosing $q^{\prime}=\frac{2}{q}$, with $\frac{1}{p^{\prime}}+\frac{1}{q^{\prime}}=1$, and by (6.3),

$$
\begin{aligned}
\|\eta\|_{L_{q}} & =\left(\int_{B_{2 h}(x)}|\eta|^{q} d x\right)^{\frac{1}{q}} \leq C\left(\int_{B_{2 h}(x)} 1 d x\right)^{\frac{1}{p^{\prime} q}}\left(\int_{B_{2 h}(x)}|\eta|^{q q^{\prime}} d x\right)^{\frac{1}{q q^{\prime}}} \\
& \leq C h^{\frac{2}{p^{\prime} q}}\|\eta\|_{L_{2}} \leq C h^{\frac{2}{p^{\prime} q}-1} .
\end{aligned}
$$

Note that $2 / p^{\prime} q=1-2 / \ln (1 / h)$. Thus, $h^{\left(2 / p^{\prime} q\right)-1}=h^{-2 / \ln (1 / h)} \leq C$. Hence, we can conclude that $\|\eta\|_{-1} \leq C \ln (1 / h)$. We thus have the desired inequality.

Lemma 6.2. Let $g^{x}$ and $g_{h}^{x}$ satisfy (6.2) and (6.4). Then

$$
\left\|g^{x}-g_{h}^{x}\right\|_{W_{1}^{1}(\Omega)} \leq C h\left(\ln \frac{1}{h}\right)^{3 / 2} \text {. }
$$


Proof. Let $M>>1$ be a constant which will be chosen later on to be sufficiently large. For convenience we shall choose $M$ to begin with so that for some integer $J$

$$
M h=2^{-J} .
$$

Note that since $M>1$

$$
J=\ln _{2} \frac{1}{M}+\ln _{2} \frac{1}{h} \leq \ln _{2} \frac{1}{h} .
$$

Then, using Cauchy-Schwartz inequality, Theorem 4.1, and (6.3),

$$
\begin{aligned}
\left\|g^{x}-g_{h}^{x}\right\|_{W_{1}^{1}(\Omega)} & =\left\|g^{x}-g_{h}^{x}\right\|_{W_{1}^{1}\left(B_{M h}(x)\right)}+\sum_{j=0}^{J}\left\|g^{x}-g_{h}^{x}\right\|_{W_{1}^{1}\left(\Omega_{j}\right)} \\
& \leq C M h \cdot h \mid g^{x}\left\|_{W_{2}^{2}(\Omega)}+\sum_{j=0}^{J} d_{j}\right\| g^{x}-g_{h}^{x} \|_{W_{2}^{1}\left(\Omega_{j}\right)} \\
& \leq C M h+\sum_{j=0}^{J} d_{j}\left\|g^{x}-g_{h}^{x}\right\|_{W_{2}^{1}\left(\Omega_{j}\right)} .
\end{aligned}
$$

By Theorem 5.1 with $\chi=\left(g^{x}\right)_{I}$ and the approximation property (2.9), we have

$$
\begin{gathered}
\left\|g^{x}-g_{h}^{x}\right\|_{W_{2}^{1}\left(\Omega_{j}\right)} \\
\leq C\left(\left\|g^{x}-\chi\right\|_{W_{2}^{1}\left(\Omega_{j}^{\prime}\right)}+\frac{\left\|g^{x}-\chi\right\|_{L_{2}\left(\Omega_{j}^{\prime}\right)}}{d_{j}}+\frac{\left\|g^{x}-g_{h}^{x}\right\|_{L_{2}\left(\Omega_{j}^{\prime}\right)}}{d_{j}}\right) \\
+C\left(h^{3}\left\|g^{x}-\chi\right\|_{W_{2}^{2}\left(\Omega_{j}^{\prime}\right)}^{h}+h\left\|g^{x}-\chi\right\|_{W_{2}^{1}(\Omega)}\right. \\
\left.+\left\|g^{x}-\chi\right\|_{L_{2}(\Omega)}+\left\|g^{x}-g_{h}^{x}\right\|_{L_{2}(\Omega)}\right) \\
\leq C\left(h\left\|g^{x}\right\|_{W_{2}^{2}\left(\Omega_{j}^{\prime \prime}\right)}+\frac{1}{d_{j}}\left\|g^{x}-g_{h}^{x}\right\|_{L_{2}\left(\Omega_{j}^{\prime}\right)}\right)+C h^{2}\left\|g^{x}\right\|_{W_{2}^{2}(\Omega)} .
\end{gathered}
$$

By definition of $g^{x}$, it is an $A$-harmonic function on $\Omega_{j}^{\prime \prime}$, i.e., $A\left(g^{x}, \psi\right)=0$ for $\operatorname{supp}(\psi) \subset \Omega_{j}^{\prime \prime}$, for $j=0, \ldots, J$. Thus, by local regularity estimates,

$$
\left\|g^{x}\right\|_{W_{2}^{2}\left(\Omega_{j}^{\prime \prime}\right)} \leq \frac{C}{d_{j}}\left\|g^{x}\right\|_{W_{2}^{1}\left(\Omega_{j}^{\prime \prime \prime}\right)} .
$$

Here,

$$
\begin{aligned}
\left\|g^{x}-g_{h}^{x}\right\|_{W_{1}^{1}(\Omega)} & \leq C M h+\sum_{j=0}^{J} d_{j}\left\|g^{x}-g_{h}^{x}\right\|_{W_{2}^{1}\left(\Omega_{j}\right)} \\
& \leq C M h+C\left(\sum_{j=0}^{J} d_{j} h\left\|g^{x}\right\|_{W_{2}^{2}\left(\Omega_{j}^{\prime \prime}\right)}\right)+C \sum_{j=0}^{J}\left\|g^{x}-g_{h}^{x}\right\|_{L_{2}\left(\Omega_{j}^{\prime}\right)} \\
& \leq C M h+C \sum_{j=0}^{J} h\left\|g^{x}\right\|_{W_{2}^{1}\left(\Omega_{j}^{\prime \prime \prime}\right)}+C\left(\ln \frac{1}{h}\right)^{1 / 2}\left\|g^{x}-g_{h}^{x}\right\|_{L_{2}(\Omega)} \\
& \leq C h \ln \frac{1}{h}+C h\left(\ln \frac{1}{h}\right)^{1 / 2}\left\|g^{x}\right\|_{W_{2}^{1}(\Omega)} \\
& \leq C h\left(\ln \frac{1}{h}\right)^{3 / 2}
\end{aligned}
$$


We shall use the above to prove our main result of this section.

Theorem 6.3. For any fixed $x \in \bar{\Omega}$,

$$
\left|\left(u-u_{h}\right)(x)\right| \leq C h\left(\ln \frac{1}{h}\right)^{3 / 2}\left(\left\|u-u_{I}\right\|_{W_{\infty}^{1}}+h\left\|u-u_{I}\right\|_{2}^{h}\right) .
$$

Proof. For any $\psi \in W_{h}$, the triangle inequality, inverse inequality (2.11), and (2.10) yield

$$
\begin{aligned}
\left|\left(u-u_{h}\right)(x)\right| \leq & |(u-\psi)(x)|+C h^{-1}\left\|\psi-u_{h}\right\|_{L_{2}\left(B_{2 h}(x)\right)} \\
\leq & |(u-\psi)(x)| \\
& +C h^{-1}\left(\|\psi-u\|_{L_{2}\left(B_{2 h}(x)\right)}+\left\|u-u_{h}\right\|_{L_{2}\left(B_{2 h}(x)\right)}\right) \\
\leq & C\|u-\psi\|_{L_{\infty}\left(B_{2 h}(x)\right)}+C h^{-1}\left\|u-u_{h}\right\|_{L_{2}\left(B_{2 h}(x)\right)} \\
\leq & C h\|u\|_{W_{\infty}^{1}}+C h^{-1}\left\|u-u_{h}\right\|_{L_{2}\left(B_{2 h}(x)\right) .}
\end{aligned}
$$

By writing $\left(u-u_{h}\right)=\left(u-u_{I}\right)+\left(u_{I}-u_{h}\right)$, we obtain

$$
\left|\left(u-u_{h}\right)(x)\right| \leq C h\left\|u-u_{I}\right\|_{W_{\infty}^{1}}+C h^{-1}\left\|u-u_{h}\right\|_{L_{2}\left(B_{2 h}(x)\right)} .
$$

By definition of $\eta, g^{x}$ and $B(\cdot, \cdot)$ with the fact $\left[g_{\nu}^{x}\right]=0$, we have

$$
\begin{aligned}
h^{-1} & \left\|u-u_{h}\right\|_{L_{2}\left(B_{2 h}\right)} \\
& =\left(u-u_{h}, \eta\right)=A\left(u-u_{h}, g^{x}\right) \\
& =A\left(u-u_{h}, g^{x}\right)+A\left(u-u_{h}, T_{h}(\beta-1) g^{x}\right)-A\left(u-u_{h}, T_{h}(\beta-1) g^{x}\right) \\
& =B\left(u-u_{h}, g^{x}\right)-h^{2} \sum_{\tau} h_{\tau}^{2}\left(L\left(u-u_{h}\right), L g^{x}\right)_{\tau}-A\left(u-u_{h}, T_{h}(\beta-1) g^{x}\right) \\
& =I_{1}+I_{2}+I_{3} .
\end{aligned}
$$

By the orthogonal property (3.3),

$$
\begin{aligned}
I_{1}= & B\left(u-u_{h}, g^{x}\right) \\
= & B\left(u-u_{h}, g^{x}-g_{h}^{x}\right)=B\left(u-\chi, g^{x}-g_{h}^{x}\right) \\
= & A\left(u-\chi, g^{x}-g_{h}^{x}\right)+A\left(u-\chi, T_{h}(\beta-1)\left(g^{x}-g_{h}^{x}\right)\right) \\
& +h^{2} \sum_{\tau} h_{\tau}^{2}\left(L(u-\chi), L\left(g^{x}-g_{h}^{x}\right)\right)_{\tau}+h^{2} \sum_{e} h_{\tau(e)} \int_{e}\left[(u-\chi)_{\nu}\right]\left[\left(g^{x}-g_{h}^{x}\right)_{\nu}\right] d s \\
= & J_{1}+J_{2}+J_{3}+J_{4},
\end{aligned}
$$

for any $\chi \in W_{h}$. For the simple presentation of our result, we choose $\chi=u_{I}$.

By Lemma 6.2.

$$
\begin{aligned}
\left|J_{1}\right| & =\left|A\left(u-u_{I}, g^{x}-g_{h}^{x}\right)\right| \leq C\left\|u-u_{I}\right\|_{W_{\infty}^{1}}\left\|g^{x}-g_{h}^{x}\right\|_{W_{1}^{1}} \\
& \leq C h\left(\ln \frac{1}{h}\right)^{3 / 2}\left\|u-u_{I}\right\|_{W_{\infty}^{1}} .
\end{aligned}
$$

By (2.21), Theorem 4.3, (6.3), and approximation property (2.9),

$$
\begin{aligned}
\left|J_{2}\right| & =\left|A\left(u-u_{I}, T_{h}(\beta-1)\left(g^{x}-g_{h}^{x}\right)\right)\right| \leq C\left\|u-u_{I}\right\|_{1}\left\|T_{h}(\beta-1)\left(g^{x}-g_{h}^{x}\right)\right\|_{1} \\
& \leq C\left\|u-u_{I}\right\|_{1}\left\|g^{x}-g_{h}^{x}\right\|_{0} \leq C h^{2}\left\|u-u_{I}\right\|_{1}\left\|g^{x}\right\|_{2} \leq C h\left\|u-u_{I}\right\|_{1} .
\end{aligned}
$$

By Lemma 4.2 and (6.3),

$$
\begin{aligned}
\left|J_{3}\right| & =\left|h^{2} \sum_{\tau} h_{\tau}^{2}\left(L\left(u-u_{I}\right), L\left(g^{x}-g_{h}^{x}\right)\right)_{\tau}\right| \leq C h^{2}\left\|u-u_{I}\right\|_{2}^{h}\left\|g^{x}-g_{h}^{x}\right\|_{2}^{h} \\
& \leq C h^{2}\left\|u-u_{I}\right\|_{2}^{h} h^{2}\left\|g^{x}\right\|_{2} \leq C h^{3}\left\|u-u_{I}\right\|_{2}^{h} .
\end{aligned}
$$


By the trace inequality (3.4), Lemma 4.2, approximation property (2.9), and (6.3),

$$
\begin{aligned}
\left|J_{4}\right|= & \left|h^{2} \sum_{e} h_{\tau(e)} \int_{e}\left[\left(u-u_{I}\right)_{\nu}\right]\left[\left(g^{x}-g_{h}^{x}\right)_{\nu}\right] d s\right| \\
\leq & C h^{3} \sum_{\tau}\left(\left(\frac{1}{\sqrt{h}}\left\|u-u_{I}\right\|_{1, \tau}+\sqrt{h}\left\|u-u_{I}\right\|_{2, \tau}\right)\right. \\
& \left.\cdot\left(\frac{1}{\sqrt{h}}\left\|g^{x}-g_{h}^{x}\right\|_{1, \tau}+\sqrt{h}\left\|g^{x}-g_{h}^{x}\right\|_{2, \tau}\right)\right) \\
\leq & C h^{2} \sum_{\tau}\left(\left\|u-u_{I}\right\|_{1, \tau}+h\left\|u-u_{I}\right\|_{2, \tau}\right)\left(\left\|g^{x}-g_{h}^{x}\right\|_{1, \tau}+h\left\|g^{x}-g_{h}^{x}\right\|_{2, \tau}\right) \\
\leq & C h^{2}\left(\left\|u-u_{I}\right\|_{1}\left(\left\|g^{x}-g_{h}^{x}\right\|_{1}+h\left\|g^{x}-g_{h}^{x}\right\|_{2}^{h}\right)\right) \\
& +C h^{2}\left(h\left\|u-u_{I}\right\|_{2}^{h}\left(\left\|g^{x}-g_{h}^{x}\right\|_{1}+h\left\|g^{x}-g_{h}^{x}\right\|_{2}^{h}\right)\right) \\
\leq & C h^{2}\left\|u-u_{I}\right\|_{1} \cdot h\|g\|_{2}+C h^{3}\left\|u-u_{I}\right\|_{2}^{h} \cdot h\|g\|_{2} \\
\leq & C h^{2}\left\|u-u_{I}\right\|_{1}+C h^{3}\left\|u-u_{I}\right\|_{2}^{h} .
\end{aligned}
$$

For $I_{2}$, using (6.3) and Lemma 4.2 ,

$$
\begin{aligned}
\left|I_{2}\right| & =\left|h^{2} \sum_{\tau} h_{\tau}^{2}\left(L\left(u-u_{h}\right), L g^{x}\right)_{\tau}\right| \leq C h^{4}\left\|u-u_{h}\right\|_{2}^{h}\left\|g^{x}\right\|_{2} \\
& \leq C h^{3}\left\|u-u_{h}\right\|_{2}^{h} \leq C h^{2}\left(\left\|u-u_{I}\right\|_{1}+h\left\|u-u_{I}\right\|_{2}^{h}\right) .
\end{aligned}
$$

For $I_{3}$,

$$
\begin{aligned}
I_{3} & =-A\left(u-u_{h}, T_{h}(\beta-1) g^{x}\right) \\
& =-A\left(u-u_{h},\left(T_{h}-T\right)(\beta-1) g^{x}\right)-A\left(u-u_{h}, T(\beta-1) g^{x}\right) .
\end{aligned}
$$

Using the definition of $T$ (2.7) and $T_{h}$ (2.15),

$$
\begin{aligned}
& A\left(u-u_{h}, T(\beta-1) g^{x}\right) \\
&=\left(\nabla\left(u-u_{h}\right), \nabla T(\beta-1) g^{x}\right)+\left(\beta\left(u-u_{h}\right), T(\beta-1) g^{x}\right) \\
&=\left(u-u_{h},(\beta-1) g^{x}\right)-\left(u-u_{h}, T(\beta-1) g^{x}\right) \\
& \quad+\left(\beta\left(u-u_{h}\right), T(\beta-1) g^{x}\right) \\
&=\left(u-u_{h},(\beta-1) g^{x}\right)+\left((\beta-1)\left(u-u_{h}\right), T(\beta-1) g^{x}\right) .
\end{aligned}
$$

Thus, using (2.17), (2.16), Theorem 4.1, and Lemma 6.1,

$$
\begin{aligned}
\left|I_{3}\right| \leq & \left\|u-u_{h}\right\|_{1}\left\|\left(T-T_{h}\right)(\beta-1) g^{x}\right\|_{1} \\
& \quad+\left\|u-u_{h}\right\|_{0}\left\|g^{x}\right\|_{1}+\left\|u-u_{h}\right\|_{0}\left\|T(\beta-1) g^{x}\right\|_{1} \\
\leq & C h\left\|u-u_{h}\right\|_{1}\left\|g^{x}\right\|_{1} \\
\leq & C h\left(\ln \frac{1}{h}\right)\left(\left\|u-u_{I}\right\|_{1}+h\left\|u-u_{I}\right\|_{2}^{h}\right) .
\end{aligned}
$$

By combining $I_{1}, I_{2}$, and $I_{3}$ in (6.6), we can conclude that

$$
\left|\left(u-u_{h}\right)(x)\right| \leq C h\left(\ln \frac{1}{h}\right)^{3 / 2}\left(\left\|u-u_{I}\right\|_{W_{\infty}^{1}}+\left\|u-u_{I}\right\|_{1}+h\left\|u-u_{I}\right\|_{2}^{h}\right) .
$$

Since $\left\|u-u_{I}\right\|_{1} \leq\left\|u-u_{I}\right\|_{W_{\infty}^{1}}$, we obtain the statement of the theorem.

For the error estimates for $\nabla\left(u-u_{h}\right)$ we can follow the argument given in [13]. We state our theorem without proof. 
Theorem 6.4. For any fixed $x \in \bar{\Omega}$,

$$
\left|\nabla\left(u-u_{h}\right)(x)\right| \leq C\left(\ln \frac{1}{h}\right)^{3 / 2}\left(\left\|u-u_{I}\right\|_{W_{\infty}^{1}}+h\left\|u-u_{I}\right\|_{2}^{h}\right) .
$$

\section{NumERICAL RESUltS}

We include here numerical results for a test problem. Let $\Omega \subset \mathbb{R}^{2}$ be a square domain with corners $(0,0),(1,0),(1,1),(0,1)$. Consider the following model problem:

$$
\begin{array}{rll}
-\triangle u-30 u=f & & \text { in } \Omega, \\
u=0 & & \text { on } \partial \Omega,
\end{array}
$$

with the exact solution

$$
u=\left(x-x^{2}\right) \sin (\pi y) .
$$

Note that we choose our model problem so that the problem is indefinite.

For approximation, we use a regular grid of triangles and a subspace $W_{h}$ of continuous piecewise linear functions defined with respect to this triangulation. Specifically, we portion the square into $N \times N$ smaller squares and then into two triangles by connecting the lower left and upper right corner vertices. Functions in $W_{h}$ vanish on $\partial \Omega$. We present the convergence behavior based on (3.2) in Table 7.1. A seven-point quadrature rule [16] which integrates exactly polynomials of up to degree five was used for the computation.

At the end of Section 3, we discussed using different powers of $h$ in our bilinear form, i.e., we can take our bilinear form as follows:

$$
\begin{aligned}
B(u, v)=A(u, v)+ & A\left(u, T_{h}(\beta-1) v\right) \\
& +h^{n} \sum_{\tau} h_{\tau}^{2}(L u, L v)_{\tau}+h^{n} \sum_{e} h_{\tau(e)} \int_{e}\left[u_{\nu}\right]\left[v_{\nu}\right] d s,
\end{aligned}
$$

where $n \geq 1$. We include numerics for $n=0,2,4,6,8, \infty$ in Table 7.2 for $H^{1}$-norm with our model problem. Note that $n=0$ corresponds to the method proposed by Bramble et al. [4 and $n=2$ corresponds to the method based on (3.2). We denote $n=\infty$ when we remove the last two terms, and the least-squares method coincides with the Galerkin method as explained in [4, Remark 3.2].

The numerics confirms Theorems 4.1 and 6.3 in this paper and shows the improvement due to our modification. We note that the least-squares method behaves better than the Galerkin method for $h=\frac{1}{4}, \frac{1}{8}$.

TABle 7.1. Convergence behavior.

\begin{tabular}{c|c|c|c|c}
\hline$h$ & Discrete $L_{2}$ error & rate & Maximum norm error & rate \\
\hline $1 / 4$ & 0.0595 & - & 0.1111 & - \\
$1 / 8$ & 0.0037 & 4.01 & 0.0113 & 3.30 \\
$1 / 16$ & 0.0021 & 0.82 & 0.0058 & 0.96 \\
$1 / 32$ & $6.1190 e-4$ & 1.78 & 0.0016 & 1.86 \\
$1 / 64$ & $1.6255 e-4$ & 1.91 & $4.2759 e-4$ & 1.90 \\
\hline
\end{tabular}


TABLE 7.2. Convergence behavior for varying power of $h$ for the $H^{1}$-norm.

\begin{tabular}{c|c|c|c|c|c|c}
\hline$h$ & $n=0$ & $n=2$ & $n=4$ & $n=6$ & $n=8$ & $n=\infty$ \\
\hline $1 / 4$ & 0.4542 & 0.3022 & 0.3355 & 0.4176 & 0.4239 & 0.4244 \\
$1 / 8$ & 0.3916 & 0.1182 & 0.1338 & 0.1341 & 0.1341 & 0.1341 \\
$1 / 16$ & 0.2560 & 0.0593 & 0.0599 & 0.0599 & 0.0599 & 0.0599 \\
$1 / 32$ & 0.1103 & 0.0291 & 0.0291 & 0.0291 & 0.0291 & 0.0291 \\
$1 / 64$ & 0.0358 & 0.0144 & 0.0144 & 0.0144 & 0.0144 & 0.0144 \\
\hline
\end{tabular}

\section{ACKNOWLEDGMENTS}

The author would like to thank Professors Lars Wahlbin and Alfred Schatz for helpful conversations and guidance.

\section{REFERENCES}

1. A. K. Aziz, R. B. Kellogg and A. B. Stephens, Least-squares methods for elliptic systems, Math. Comp., 44(1985), pp. 53-70.

2. P. B. Bochev and M. D. Gunzburger, Analysis of least-squares finite element methods for the Stokes equations, Math. Comp., 63(1994), pp. 479-506.

3. J. H. Bramble, R. D. Lazarov and J. E. Pasciak, A least-squares approach based on a discrete minus one inner product for first order systems, Math. Comp., 66(1997), pp. 935-955.

4. J. H. Bramble, R. D. Lazarov and J. E. Pasciak, Least squares for 2nd order elliptic problems, Comput. Methods Appl. Mech. Engrg, 152(1998), no. 1-2, pp. 195-210.

5. J. H. Bramble and A. H. Schatz, Least squares for $2 m$ th order elliptic boundary-value problems, Math. Comp., 25(1971), pp. 1-32.

6. S. C. Brenner and L. R. Scott, The Mathematical Theory of Finite Element Methods, Springer, 1994.

7. Z. Cai, R. Lazarov, T. A. Manteuffel, and S. F. McCormick, First-order system least squares for second-order partial differential equations: part I, SIAM J. Numer. Anal., 31(1994), pp. 1785-1799.

8. Z. Cai, T. A. Manteuffel, and S. F. McCormick, First-order system least squares for secondorder partial differential equations: part II, SIAM J. Numer. Anal., 34(1997), pp. 1727-1741.

9. P. Grisvard, Elliptic Problems in Nonsmooth Domains, Pitman, Boston, 1985.

10. J. A. Nitsche and A. H. Schatz, Interior estimates for Ritz-Galerkin methods, Math. Comp., 28(1974), pp. 937-958.

11. A. I. Pehlivanov, G. F. Carey, and P. S. Vassilievski, Least-squares mixed finite element methods for non-selfadjoint elliptic problems: I. Error extimates, Numer. Math. 72(1996), pp. 501-522.

12. A. H. Schatz, An observation concerning Ritz-Galerkin methods with indefinite bilinear forms, Math. Comp., 28(1974), pp. 159-164.

13. A. H. Schatz, Pointwise error estimates for the finite element method and asymptotic error expansion inequalities for the finite element method on irregular grids: Part I. Golobal Estimates, Math. Comp., 67(1998), pp. 877-899.

14. A. H. Schatz and L. B. Wahlbin, Interior maximum norm estimates for finite element methods, Math. Comp., 64(1995), pp. 907-928.

15. L. R. Scott and S, Zhang, Finite element interpolation of non-smooth functions satisfying boundary conditions, Math. Comp., 54(1990), pp. 483-493.

16. G. Strang and G. J. Fox, An Analysis of the Finite Element Method, Prentice-Hall, Inc., Englewood Cliffs, NJ, 1973.

17. L. B. Wahlbin, Superconvergence in Galerkin Finite Element Methods, Lecture Notes in Mathematics 1605, 1995.

Department of Mathematics, Purdue University, 150 N. University Street, West LAFAYETTE, INDiANA 47907-2067

E-mail address: jku@math.purdue.edu 